\title{
Evolution and prognosis of breast osteosarcoma: A case report
}

\author{
WANFENG GUO ${ }^{1}$, YANG CAO $^{1}$, GUANGJU TENG $^{2}$, JIN LIU $^{1}$ and JIANBIN SU ${ }^{1}$ \\ ${ }^{1}$ Department of Health Care and Medicine, The Affiliated Hospital of the Chinese Academy of Military Medical Sciences, \\ Beijing 100071; ${ }^{2}$ Department of Non-Infectious Liver Disease, 302 Hospital of the People's Liberation Army, \\ Beijing 100039, P.R. China
}

Received November 27, 2014; Accepted October 14, 2015

DOI: 10.3892/ol.2015.3921

\begin{abstract}
Breast osteosarcoma is a rare malignancy of unknown etiology, with no standard adjuvant treatment at present. The prognosis of the disease is poor, and it has a high propensity for recurrence and metastasis. The current report presents the case of a 52-year-old woman, in whom adenomyoepithelioma gradually developed into breast osteosarcoma following three separate surgeries. The patient survived for 41 months from the initial lesion occurrence and resection in the left breast; during this time, she underwent surgery and chemotherapy (liposomal doxorubicin and cisplatin) for the treatment of disease recurrence and lung metastasis, along with molecular-targeted therapy (sunitinib). However, the patient eventually succumbed to respiratory failure due to progressive disease. The present case underwent a clear pathological transformation process, and may provide a basis for an improved understanding of the clinical characteristics and treatment of breast osteosarcoma.
\end{abstract}

\section{Introduction}

Breast osteosarcoma is a rare disease that accounts for $\sim 0.1 \%$ of all breast malignancies (1). The etiological mechanisms of breast osteosarcoma remain to be elucidated. It is thought that the disease may originate from mammary stromal mesenchymal stem cells or derive from previous metaplastic mammary lesions (2). However, to date, few cases of this disease have been reported in the literature (3). Over a period of 18 years, Jernstorm et al (4) observed only one case of osteosarcoma in 3,319 cases of mammary carcinoma. Treatment for localized disease includes complete surgical removal of the tumor with an adequate margin, whereas chemotherapy is the main treatment for metastatic osteosarcoma (5). To the best of our knowledge, there is no standard adjuvant treatment for breast osteosarcoma at present. The prognosis of breast

Correspondence to: Dr Wanfeng Guo, Department of Health Care and Medicine, The Affiliated Hospital of the Chinese Academy of Military Medical Sciences, 8 Dongda Street, Beijing 100071, P.R. China

E-mail: guowanfeng@hotmail.com

Key words: breast osteosarcoma, myositis ossificans, evolution, prognosis osteosarcoma is poor, and the disease exhibits a high propensity for recurrence and metastasis (6,7).

The current report presents the case of a patient in whom adenomyoepithelioma gradually developed into breast osteosarcoma. The patient's disease progressed gradually and exploratory treatment with sunitinib; therefore the present case may provide a basis for an improved understanding of the clinical characteristics and treatment of breast osteosarcoma. Written informed consent was obtained from the patient's family.

\section{Case report}

The patient was a 52-year-old woman with no family history of hereditary cancer. A lesion was previously identified in the left breast during a regular physical examination in March 2006. The patient initially disregarded this lesion, however, the lesion gradually increased in size. Subsequently, the patient visited Zhongshan Hospital (Shanghai, China) on November 16, 2006. On December 2, 2006, a 1.7x1.5 cm tumor was resected from the upper outer quadrant of the left breast at Zhongshan Hospital. Postoperative pathological analysis of the resected specimen revealed an adenomyoepithelioma with malignant myoepithelial transformation (low-grade malignancy). The following immunohistochemical (IHC) scoring system was used, based on the percentage of immunoreactive cells and staining intensity $[<25 \%(-), 25-50 \%(+), 50-75 \%(++)$ and $>75 \%(+++)$ ], staining intensity [no color (-), light color (+), dark color $(++)]$ and the location of immunoreactivity (epithelial or focal) (8). The tumor exhibited the following IHC characteristics: Cytokeratin (CK; broad-spectrum) $(+++)$; smooth muscle actin (SMA) (++); CK5/6 (-); S-100 (-); CK7 (epithelial ++); vimentin (VIM) (+++); estrogen receptor (+); cluster of differentiation (CD)34 (-); progesterone receptor (partial +); Ki-67 (40\%); c-ErbB-2 (epithelial +); hematopoietic cell kinase (-) and CD117 (focal +).

The patient developed disease recurrence 1 month later, and the tumor subsequently continued to increase in size (Fig. 1). On March 23, 2007, a tumor was resected from the upper outer quadrant of the left breast at Zhongshan Hospital. Pathological analysis of the tumor revealed heterotopic ossification (myositis ossificans). The following IHC results were obtained: VIM (+); SMA (partial +); S-100 (weak +); CD117 (+); desmin (-); pan-CK (AE1/AE3) (-); epithelial membrane antigen (-); CD99 (-); high molecular weight CK (34ßE12) (-); CD34 (-) and multinucleated giant cells (anti-CD68/KP1) (+). 


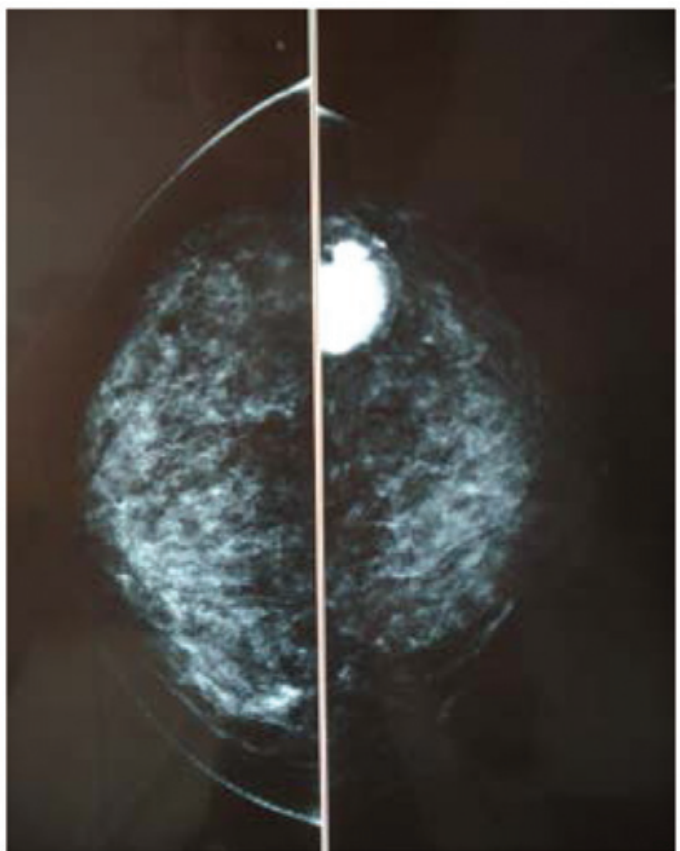

Figure 1. Mammography reveals large densely calcified mass in the left breast.

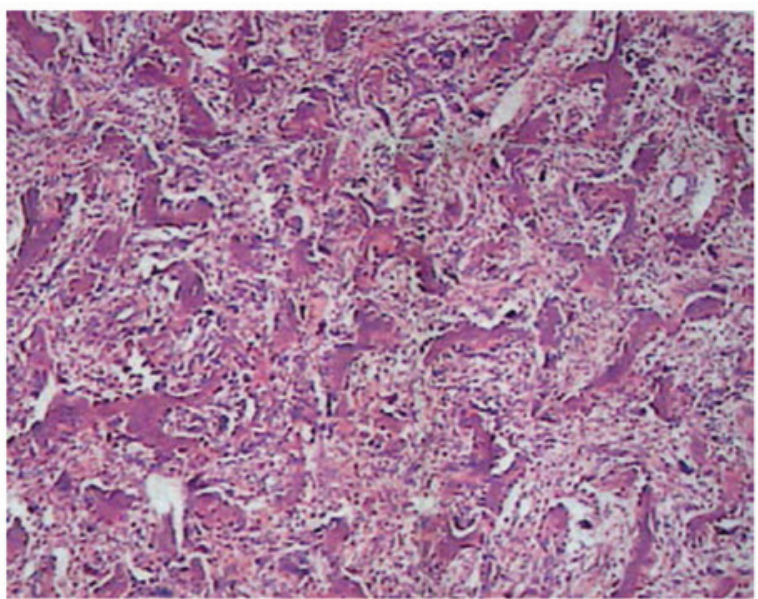

Figure 2. Photomicrograph revealing a high-grade osteosarcoma (hematoxylin-eosin stain; magnification, x150).

On August 28, 2007, the patient presented to the Cancer Hospital of Beijing (Beijing, China) with an additional relapse, and extensive local tumor resection was performed in the upper outer quadrant of the left breast. Postoperative pathological analysis revealed a well-differentiated osteosarcoma (Fig. 2). The patient did not receive any additional treatment at this time.

Positron emission tomography/computed tomography was conducted on June 25, 2008 and revealed patchy consolidation in the lungs; extrapulmonary metastases could not be excluded (Fig. 3). A total of two chemotherapy cycles, comprising $60 \mathrm{mg}$ of liposomal doxorubicin (day 1) and $50 \mathrm{mg}$ of cisplatin (days 1-3), were administered at the Cancer Hospital of Beijing, commencing on August 11 and September 9, 2008. The response evaluation (9) was observed to be stable disease (SD). However, the patient experienced grade III bone marrow suppression and grade I gastrointestinal reactions (10), and refused to undergo additional chemotherapy.

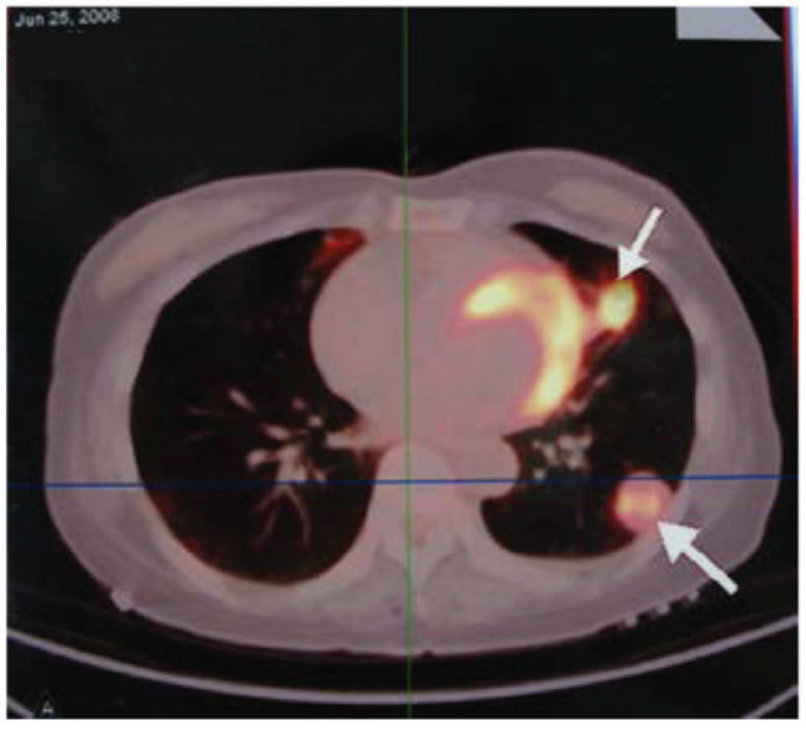

Figure 3. Positron emission tomography-computed tomography scan of the chest, revealing two masses in the left lung.

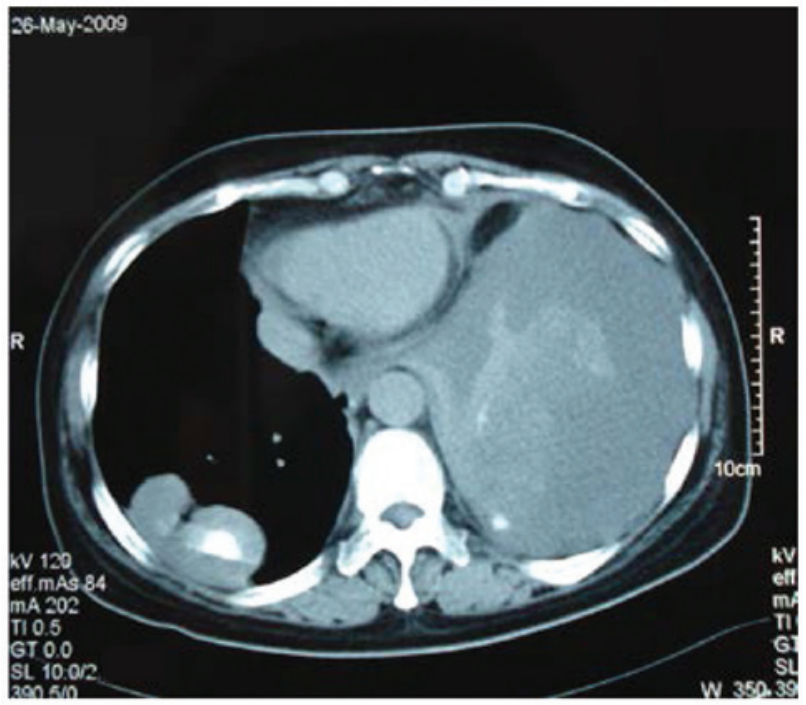

Figure 4. Computed tomography scan of the chest, revealing a large heterogeneous mass in left thoracic cavity and two nodules containing a densely calcified mass in the right thoracic cavity.

In April 2009, the disease progressed and pleural effusion occurred. Oral sunitinib (37.5 mg) was administered once daily at the Affiliated Hospital of the Chinese Academy of Military Medical Sciences (Beijing,China), according to a 4-week treatment plan. The patient exhibited diarrhea and grade II platelet reduction (10). The response evaluation was observed to be progressive disease. On May 26, 2009, the patient exhibited increased chest congestion accompanied by suffocation, and was admitted to the emergency room to undergo a pleural puncture and chest drainage. Following this procedure, $3,500 \mathrm{ml}$ of reddish fluid was extracted, and the symptoms of chest congestion and suffocation improved.

The patient refused additional chemotherapy and accepted palliative care, which included treatment with analgesics and Traditional Chinese medicine. The lesion continued to increase in size (Fig. 4), and the patient's condition 
worsened over time. She succumbed to respiratory failure in August 2009.

\section{Discussion}

Breast osteosarcoma, a type of extraskeletal osteosarcoma, is a rare disease $(1,6,7)$. It may originate from mammary stromal mesenchymal stem cells, or be derived from a fibroadenoma or phyllodes tumor (2). The etiology of breast osteosarcoma remains to be elucidated, and the disease typically occurs following a previous trauma, such as breast radiotherapy $(11,12)$.

Breast osteosarcoma occurs more frequently in middle-aged and elderly women; patients range in age from 22-82 years, with a median age of 62 years. Clinically, diagnoses of breast osteosarcoma are confirmed primarily by pathological analysis, specifically through IHC comparison with carcinosarcoma, bone cancer or breast osteomalacia (7).

Surgery is the typical treatment method for breast osteosarcoma $(7,11)$. Tumors have been observed to be resistant to radiation, and there is no evidential basis for the efficacy of chemotherapy (7,13). Silver et al (7) observed a median survival time of 23 months and 5-year survival rate of $39 \%$ for patients with breast osteosarcoma following surgical treatment. The reported 1-year recurrence rate of extraskeletal osteosarcomas is $\sim 43 \%$ (14). The prognostic factors of breast osteosarcoma may be associated with tumor size, grade and status, such as margin status (7,15). Additionally, Zhao et al (16) proposed that prognosis is improved for breast osteosarcoma cases that contain a rich cartilage matrix and fibroblast cells.

The patient in the present case underwent several postoperative pathological examinations that produced inconsistent results. However, this was a gradually evolving process. Initially, the patient was diagnosed with adenomyoepithelioma with malignant myoepithelial transformation; 3 months later, her condition had progressed to heterotopic ossification (myositis ossifications). Disease recurrence was detected at 5 months after the second surgery, and this tumor was diagnosed as a well-differentiated osteosarcoma. This is consistent with the hypothesis that breast osteosarcoma represents an occurrence of metaplasia following breast trauma and ongoing ossification of the fibrous components.

Recurrence and metastasis are prevalent in cases of breast osteosarcoma. For example, Silver et al (7) reported the occurrence of metastases in $42 \%$ of breast osteosarcoma patients. These events primarily occur in the form of blood metastases, and are typically accompanied by pulmonary metastases $(6,7)$. The current patient presented with clear pulmonary metastases.

Chemotherapeutic drugs (including, doxorubicin, cyclophosphamide and cisplatin) may prolong survival in patients exhibiting primary osteosarcoma; however, this has not been demonstrated in cases of breast osteosarcoma $(11,16,17)$. In the present case, liposomal doxorubicin and cisplatin chemotherapy were selected for treatment of the patient, and an SD response evaluation was achieved. However, this chemotherapeutic regimen caused significant adverse events and inhibited medullary hematopoiesis. The patient refused additional chemotherapy, and the use of sunitinib, which is a multimolecular tyrosine kinase inhibitor that has exhibited promising results for the treatment of soft tissue sarcoma $(18,19)$. The patient was subsequently administered $37.5 \mathrm{mg}$ of oral sunitinib once daily, according to a 4-week treatment plan; however, poor results were achieved. Multimolecular-targeted drugs may therefore be unsuitable for the treatment of breast osteosarcoma.

In summary, osteosarcoma is relatively rare in clinical practice. The histogenesis of primary osteosarcoma of the breast remains unclear, however, the results of the present case indicate that a transformation from adenomyoepithelioma may be involved. To date, no optimal treatment has been identified for patients with recurrent and metastatic osteosarcoma. Furthermore, the efficacy of chemotherapy remains unclear, and emerging molecular targeted therapies may not present a promising therapeutic option. Therefore, further research is required in order to understand its pathogenesis and effective treatments.

\section{References}

1. Adem C, Reynolds C, Ingle JN and Nascimento AG: Primary breast sarcoma: Clinicopathologic series from the Mayo Clinic and review of the literature. Br J Cancer 91: 237-241, 2004.

2. Haberthür F, Feichter GE and Torhorst JK: Sarcoma of the breast: What is the contribution of immunohistochemistry and flow cytometry? Geburtshilfe Frauenheilkd 53: 811-813, 1993 (In German).

3. Krishnamurthy A: Primary breast osteosarcoma: A diagnostic challenge. Indian J Nucl Med 30: 39-41, 2015.

4. Jernstrom P, Lindberg AL and Meland ON: Osteogenic sarcoma of the mammary gland. Am J Clin Pathol 40: 521, 1963.

5. Al Samaraee A, Angamuthu N and Fasih T: Primary breast osteosarcoma: A case report and review of literature. Scott Med J 59: e1-e4, 2014.

6. Remadi S, Doussis-Anagnostopoulu I and Mac Gee W: Primary osteosarcoma of the breast. Pathol Res Pract 191: 471-474, 1995.

7. Silver SA and Tavassoli FA: Primary osteogenic sarcoma of the breast: A clinicopathologic analysis of 50 cases. Am J Surg Pathol 22: 925-933, 1998.

8. Jacobs TW, Gown AM, Yaziji H, Barnes MJ and Schnitt SJ: Comparison of fluorscence in situ hybridization and immunohistochemistry for the evaluation of HER-2/neu in breast cancer. J Clin Oncol 17: 1974-1982, 1999.

9. Therasse P, Arbuck SG, Eisenhauer EA, et al: New guidelines to evaluate the response to treatment in solid tumors. European Organization for Research and Treatment of Cancer, National Cancer Institute of the United States, National Cancer Institute of Canada. J Natl Cancer Inst 92: 205-216, 2000.

10. Common Terminology Criteria for Adverse Events v3.0 (CTCAE), 2006. http://ctep.cancer.gov/protocolDevelopment/electronic_ applications/docs/ctcaev3.pdf.

11. Lagrange JL, Ramaioli A, Chateau MC, et al: Sarcoma after radiation therapy: Retrospective multiinstitutional study of 80 histologically confirmed cases. Radiation therapist and pathologist groups of the Fédération Nationale des Centres de Lutte Contre le Cancer. Radiology 216: 197-205, 2000.

12. Nwankwo N, Barbaryan A, Ali AM, et al: Breast osteosarcoma 29 years after radiation therapy for epithelial breast cancer. Case Rep Oncol 6: 367-372, 2013.

13. Lum WY and Jacobs L: Primary breast sarcoma. Surg Clin North Am 88: 559-570, 2008.

14. Vorobiof G, Hariparsad G, Freinkel W, Said H and Vorobiof DA: Primary osteosarcoma of the breast: A case report. Breast J 9: 231-233, 2003.

15. Blanchard DK, Reynolds CA, Grant CS and Donohue JH: Primary nonphylloides breast sarcomas. Am J Surg 186: 359-361, 2003.

16. Zhao J, Zhang X, Liu J and Li J: Primary osteosarcoma of the breast with abundant chondroid matrix and fibroblasts has a good prognosis: A case report and review of the literature. Oncol Lett 6: 745-747, 2013.

17. Szajewski M, Kruszewski WJ, Ciesielski M, et al: Primary osteosarcoma of the breast: A case report. Oncol Lett 7: 1962-1964,2014.

18. Cassier PA, Dufresne A, Fayette J, et al: Emerging drugs for the treatment of soft tissue sarcomas. Expert Opin Emerg Drugs 12: 139-153, 2007.

19. Shor AC, Agresta SV, D'Amato GZ and Sondak VK: Therapeutic potential of directed tyrosine kinase inhibitor therapy in sarcomas. Cancer Control 15: 47-54, 2008. 